\title{
BMJ Open Hip abductor muscle strength in patients after total or unicompartmental knee arthroplasty for knee osteoarthritis or avascular necrosis: a systematic review and meta-analysis protocol
}

To cite: Kvarda P, Nüesch C, Egloff C, et al. Hip abductor muscle strength in patients after total or unicompartmental knee arthroplasty for knee osteoarthritis or avascular necrosis: a systematic review and meta-analysis protocol. BMJ Open 2020;10:e038770. doi:10.1136/ bmjopen-2020-038770

- Prepublication history and additional material for this paper are available online. To view these files, please visit the journal online (http://dx.doi org/10.1136/bmjopen-2020038770).

Received 23 March 2020 Revised 30 June 2020 Accepted 03 July 2020
Check for updates

(C) Author(s) (or their employer(s)) 2020. Re-use permitted under CC BY-NC. No commercial re-use. See rights and permissions. Published by BMJ.

For numbered affiliations see end of article.

Correspondence to

Dr Peter Kvarda;

kvardamed@gmail.com

\section{ABSTRACT}

Introduction Reduced hip abductor strength may indirectly lead to changes in knee kinematics and functional impairment and has been reported in patients with patellofemoral pain and knee osteoarthritis $(\mathrm{OA})$. Limited information is available regarding hip abductor strength following total or unicompartmental knee arthroplasty (TKA/UKA). The aims of this systematic review are to synthesise the evidence of hip abductor muscle strength deficits in patients following TKA/UKA and to determine influencing factors for these deficits. Methods and analysis Embase, Medline, SportDiscus, the Web of Science Core Collection and Scopus will be searched for human-based clinical studies investigating hip abductor muscle strength after TKA/UKA for knee OA or avascular necrosis (AVN). Articles studying hip abductor strength after knee arthroplasty for posttraumatic $\mathrm{OA}$ will not be considered. No restriction on study design, prosthesis design, surgical approach, patient characteristics or severity of OA/AVN will be applied. We will search articles published between 1 January 1990 and the date of our last search. Only articles in English or German language will be considered for inclusion. Studies reporting manually measured muscle strength or measurements performed at hip abduction angles other than $0^{\circ}$ will be excluded. References will be screened by two reviewers independently. Where necessary, a third author will make the final decision. The assessment of quality and risk of bias will be performed with the modified Newcastle-Ottawa scale. Data will be extracted and presented in a tabular form. Depending on availability, comparable subgroup and meta-analyses will be conducted. Patient characteristics such as age, sex and surgical approach or rehabilitation programme will be analysed, if sufficient data are available.

Ethics and dissemination No ethics approval is required. The results will be published in a peer-reviewed journal and as conference presentation.

\section{INTRODUCTION}

Degenerative diseases of the musculoskeletal system such as osteoarthritis (OA) are one of the leading burdens on the healthcare system,

\section{Strengths and limitations of this study}

- To our knowledge, there is no published systematic review investigating hip abductor muscle strength following total or unicompartmental knee arthroplasty

- The subject and inclusion/exclusion criteria are clearly stated to obtain and present comparable data.

- Possible limitations are the restricted time period of publication and language restriction to English or German.

- Possibility of limited and heterogeneous data availability to perform a meta-analysis.

social security system and certainly on individuals. Prevalence of knee OA is estimated to be approximately $10 \%$ in men and $13 \%$ in women at the age of 60 years or older. ${ }^{1} \mathrm{OA}$ is estimated to be the fourth-leading cause of disability by the year of 2020, and the most common indication for performing knee arthroplasty. $^{23}$

Different static and dynamic biomechanical components influence the functional knee mechanics. Static elements are alignment and bony geometry. A neutral mechanical axis of the lower limb during standing passes through the centre of the tibial plateau in the frontal plane. This axis is altered in valgus or varus deformity. ${ }^{45}$ Physiologically, during the stance phase of walking, the centre of load is located over the medial condyle creating an external knee adduction moment. ${ }^{6} 7$ Ligaments and muscles of the joint form the group of dynamic stabilisers and mainly resist the adduction moment. ${ }^{7}$

As the adjacent proximal articulation, the hip joint contributes to knee 
biomechanics. The hip abductor muscles abduct the femur, facilitate pelvic stability during single leg stance and walking, and directly affect the tibiofemoral and patellofemoral joint kinematics. Moreover, the hip abductor muscle group controls the internal rotation of the femur. ${ }^{8}$ In case of hip abductor muscle strength deficit, the contralateral pelvic side drops while walking, a condition known as 'trendelenburg gait'9 because the external hip adduction moment cannot be sufficiently balanced by the internal hip abduction moment primarily generated by hip abductor muscles. This can be compensated by leaning the trunk towards the support limb and shifting the centre of mass over the support limb and reduce the hip adduction moment. ${ }^{8}$ The protective effect of greater internal hip abduction moment has also been reported in terms of reduced medial tibiofemoral OA progression. ${ }^{10}$ Moreover, patellar tracking can be also altered and cause knee pain. ${ }^{11-13}$ Isokinetic measurements have shown that hip abductor muscle weakness is present in patients with knee OA. ${ }^{14}{ }^{15}$ In a recent systematic review, Deasy et al reported hip abductor weakness in patients with knee OA. ${ }^{16}$

Current non-surgical treatment modalities aiming alleviate and control symptoms, nonetheless today the only treatment for severe knee OA is total or unicompartmental knee arthroplast (TKA/UKA). ${ }^{1718}$ Reduced hip abductor muscle strength can implicate compromised functional and performance-based outcome after TKA/UKA, and hence maintaining and strengthening of the hip abductor muscles are clinically relevant factors in patients undergoing TKA or UKA. ${ }^{19-21}$ However, to date, studies investigating hip abductor muscle strength in patients undergoing TKA or UKA are scarce. In addition to outcome evaluations, quantitative assessment of muscle strength is important to understanding factors influencing surgical outcome. In contrast to knee flexor/extensor muscle strength, the impact of hip abductor muscle strength deficit in patients with TKA/UKA is currently poorly understood. The following questions remain unanswered:

- Do patients after unilateral TKA/UKA experience a muscle strength deficit in their operated compared with their unoperated side?

- How long after TKA/UKA does this deficit persist?

- Does the strength deficit after knee arthroplasty differ between patients with total vs unicompartmental arthroplasty?

- Are hip abductor muscle strength deficits after TKA/ UKA influenced by preoperative and postoperative knee alignment, patient characteristics or rehabilitation programmes?

Therefore, the aim of the proposed systematic review is to synthesise the evidence of hip abductor muscle strength deficits in patients following TKA/ UKA and to determine influencing factors for these deficits. The results of the proposed systematic review will provide extended information for physicians in
Table 1 The Population, Intervention, Comparison, Outcome, Study design process ${ }^{24}$

\begin{tabular}{|c|c|}
\hline Item & Specification \\
\hline $\begin{array}{l}\text { Population or } \\
\text { participants and } \\
\text { conditions of interest }\end{array}$ & $\begin{array}{l}\text { Patients with OA or AVN of the knee } \\
\text { (any age, gender and severity) }\end{array}$ \\
\hline Interventions & TKA/UKA \\
\hline $\begin{array}{l}\text { Comparisons or } \\
\text { control groups }\end{array}$ & $\begin{array}{l}\text { For comparison between limbs of } \\
\text { the same subject: asymptomatic } \\
\text { contralateral hip and knee } \\
\text { For comparison between } \\
\text { patients and heathy individuals: } \\
\text { asymptomatic control subjects }\end{array}$ \\
\hline Outcomes of interest & Muscle strength of hip abductors \\
\hline Study designs & $\begin{array}{l}\text { Any study design, published } \\
\text { studies, conference abstracts to be } \\
\text { considered }\end{array}$ \\
\hline
\end{tabular}

AVN, avascular necrosis; OA, osteoarthritis; TKA/UKA, total or unicompartmental knee arthroplasty.

the interest of improving patient management and outcome.

\section{METHODS AND ANALYSES}

The protocol was developed following the Preferred Reporting Items for Systematic Reviews and Meta-Analyses Protocols (PRISMA) guideline ${ }^{22}$ (online supplementary file A). Bibliographic database searching was initiated on 19 December 2019. The review was submitted for registration prospectively in PROSPERO on 5 January 2020 and the expected completion date is 1 July $2020 .{ }^{23} \mathrm{We}$ designed the study question using the Population, Intervention, Comparison, Outcome, Study design strategy (table 1$){ }^{24}$

\section{Eligibility criteria}

Inclusion criteria

Human-based clinical studies reporting on hip abductor muscle strength after primary TKA/UKA will be considered for inclusion. Case studies will not be considered for inclusion. No other restriction regarding the study design will be applied to broadly capture all possible appropriate studies. No restriction on operative approach, prosthesis design, age and sex of the patients or severity of OA/AVN will be placed. In order to avoid capturing irrelevant, methodologically inappropriate studies the date of publication will be limited to a time period from 1 January 1990 to the date of our last search. The limit of follow-up will be set to 24 months postoperatively.

\section{Exclusion criteria}

Studies published before 1 January 1990 or in a language other than English or German, not reporting absolute values of hip abductor muscle strength or torque ratios or reporting hip abductor strength measured with handheld manometer/dynamometer or at a hip abduction 
4. Differences in hip abductor torque/torque ratio between patients after TKA/UKA and healthy control groups.

The secondary outcomes will be

1. Surgical approaches/methods of TKA/UKA.

2. Preoperative and postoperative knee alignment.

3. Patient characteristics.

4. Rehabilitation programmes after TKA/UKA.

\section{Risk of bias in individual studies}

To minimise bias, articles meeting the inclusion criteria will be assessed by two reviewers (PK and PI) independently using a modified version of the NewcasteOttawa Scale $(\mathrm{NOS})^{26}$ (online supplementary file C). According to the modified NOS, each study will be valued with 1-6 stars where higher scores indicate higher level of quality. No separate tool will be used to assess the risk of bias of randomised control trials because we do not extract estimates of treatment differences from RCTs but use these as a source for cohort data.

\section{Data synthesis}

We will extract any quantitative and descriptive data from all eligible studies according to the main outcomes (mean, median, etc), on the population (SD, IQRs, percentile), on measurements (standard errors, CI, $\mathrm{p}$ values, sample size), as well as the secondary outcomes for both purposes (systematic review and meta-analyses). Furthermore, all details specific to the review question will be extracted. If the information is available for several time points, the data will be extracted for all time points. The data will be presented in tabular format. Visual inspection of the forest plots and $\mathrm{I}^{2}$ test will be used to assess heterogeneity between studies. Depending on availability of appropriate data for comparable specific patient groups with same measurement method in different studies, metaanalyses will be performed on these data. Meta-analysis will be based on random effects and the results will be illustrated by forest plots. Where statistical pooling is not possible, the findings will be presented in narrative form. Meta regression and subgroup-specific meta analyses will be conducted to investigate the effect of time since TKA/ UKA and measurement type (isokinetic or isometric) on different outcomes. In case that outcomes are not reported directly but indirect information is available on side specific or time point-specific results, the available information will be transformed accordingly. In case of sufficient information, these analyses will be extended to patient characteristics, surgical approach, subtype of prostheses or rehabilitation programme.

\section{Meta-bias(es)}

Funnel plots will be used to asses publication bias in our meta-analysis, presenting effect sizes plotted against their SEs or precisions. To avoid subjective visual inspection of the graph, Egger's regression test will be used to assess the asymmetry. Egger's test regresses the standardised effect sizes on their precisions. In the absence of publication bias, the regression intercept is expected to be zero.

\section{Confidence in cumulative evidence}

Grading of Recommendations Assessment, Development and Evaluation system will be applied. ${ }^{27}$ The confidence of evidence of the investigated study can be downgraded according to the following factors: study limitations, inconsistency of results, indirectness of evidence, imprecision, publication bias. Criteria for upgrading are the following: large magnitude of effect, evidence of a doseresponse effect and all possible confounding factors taken into account. After the grading process, the quality of evidence for each outcome will be rated as high, moderate, low or very low.

\section{Ethics and dissemination}

This study is a protocol for a systematic review and meta-analysis. No human participants will be recruited. No ethics approval is needed. The study results will be published in a peer-reviewed journal and as conference presentation.

\section{Author affiliations}

${ }^{1}$ Department of Orthopaedics and Traumatology, University Hospital Basel, Basel, Switzerland

${ }^{2}$ Department of Orthopaedic Surgery and Traumatology, Kantonsspital Baselland, Bruderholz, Basel-Landschaft, Switzerland

${ }^{3}$ Department of Clinical Research, University of Basel, Basel, Switzerland ${ }^{4}$ Department of Biomedical Engineering, University of Basel, Basel, Switzerland ${ }^{5}$ Department of Spine Surgery, University Hospital of Basel, Basel, Switzerland ${ }^{6}$ University Medical Library, University Library of Basel, Basel, Spiegelgasse, Switzerland

\section{Twitter Peter Kvarda @Kvarda}

Contributors The following work has been developed in contribution of each coauthor. The manuscript underwent several revisions with substantial contributions provided by each coauthor. PI, PK and AM will perform this systematic review and meta-analysis. The protocol has been registered in PROSPERO by PK. The search strategy was designed by $\mathrm{CA}-\mathrm{H}$. CN reviewed the protocol and was responsible for the strategy of data synthesis. CE cooperated in study design development, as well as in manuscript editing. All authors gave final approval of the manuscript prior to submission.

Funding This study will be funded by the Department of Orthopaedics and Traumatology of the University Hospital of Basel, the Foundation for Funding Science and Education at the Department of Surgery at the University of Basel, Swiss Orthopaedics, Merian Iselin Foundation and Deutsche Arthrose-Hilfe e.V.

Disclaimer The funding sources had no involvement in any aspect of this study.

Patient and public involvement Patients and/or the public were not involved in the design, or conduct, or reporting, or dissemination plans of this research.

Patient consent for publication Not required.

Provenance and peer review Not commissioned; externally peer reviewed.

Open access This is an open access article distributed in accordance with the Creative Commons Attribution Non Commercial (CC BY-NC 4.0) license, which permits others to distribute, remix, adapt, build upon this work non-commercially, and license their derivative works on different terms, provided the original work is properly cited, appropriate credit is given, any changes made indicated, and the use is non-commercial. See: http://creativecommons.org/licenses/by-nc/4.0/.

ORCID iD

Peter Kvarda http://orcid.org/0000-0002-1623-4712 


\section{REFERENCES}

1 Zhang Y, Jordan JM. Epidemiology of osteoarthritis. Clin Geriatr Med 2010;26:355-69.

2 Woolf AD, Pfleger B. Burden of major musculoskeletal conditions. Bull World Health Organ 2003;81:646-56.

3 Carr AJ, Robertsson O, Graves S, et al. Knee replacement. Lancet 2012;379:1331-40

4 Cooke TDV, Sled EA, Scudamore RA. Frontal plane knee alignment: a call for standardized measurement. J Rheumatol 2007;34:1796-801.

5 Egloff C, Hügle T, Valderrabano V. Biomechanics and pathomechanisms of osteoarthritis. Swiss Med Wkly 2012;142:W13583.

6 Morrison JB. The mechanics of the knee joint in relation to normal walking. J Biomech 1970;3:51-61.

7 Shelburne KB, Torry MR, Pandy MG. Contributions of muscles, ligaments, and the ground-reaction force to tibiofemoral joint loading during normal gait. J Orthop Res 2006;24:1983-90.

8 Powers CM. The influence of abnormal hip mechanics on knee injury: a biomechanical perspective. J Orthop Sports Phys Ther 2010;40:42-51.

9 Gandbhir VN, Rayi A. Trendelenburg gait. Treasure Island (FL): StatPearls Publishing, 2019.

10 Chang A, Hayes K, Dunlop D, et al. Hip abduction moment and protection against medial tibiofemoral osteoarthritis progression. Arthritis Rheum 2005;52:3515-9.

11 Piva SR, Goodnite EA, Childs JD. Strength around the hip and flexibility of soft tissues in individuals with and without patellofemoral pain syndrome. J Orthop Sports Phys Ther 2005;35:793-801.

12 Ireland ML, Willson JD, Ballantyne BT, et al. Hip strength in females with and without patellofemoral pain. J Orthop Sports Phys Ther 2003;33:671-6.

13 Mascal CL, Landel R, Powers C. Management of patellofemoral pain targeting hip, pelvis, and trunk muscle function: 2 case reports. $J$ Orthop Sports Phys Ther 2003;33:647-60.

14 Costa RA, Oliveira LMde, Watanabe SH, et al. Isokinetic assessment of the hip muscles in patients with osteoarthritis of the knee. Clinics 2010;65:1253-9.

15 Hinman RS, Hunt MA, Creaby MW, et al. Hip muscle weakness in individuals with medial knee osteoarthritis. Arthritis Care Res 2010;62:1190-3.
16 Deasy M, Leahy E, Semciw Al. Hip strength deficits in people with symptomatic knee osteoarthritis: a systematic review with metaanalysis. J Orthop Sports Phys Ther 2016;46:629-39.

17 Mora JC, Przkora R, Cruz-Almeida Y. Knee osteoarthritis: pathophysiology and current treatment modalities. J Pain Res 2018;11:2189-96.

18 McGrory BJ, Weber KL, Jevsevar DS, et al. Surgical management of osteoarthritis of the knee: evidence-based guideline. JAAOS - J Am Acad Orthop Surg 2016;24.

19 Loyd BJ, Jennings JM, Judd DL, et al. Influence of hip abductor strength on functional outcomes before and after total knee arthroplasty: post hoc analysis of a randomized controlled trial. Phys Ther 2017:97:896-903.

20 Piva SR, Teixeira PEP, Almeida GJM, et al. Contribution of hip abductor strength to physical function in patients with total knee arthroplasty. Phys Ther 2011;91:225-33.

21 Alnahdi AH, Zeni JA, Snyder-Mackler L. Hip abductor strength reliability and association with physical function after unilateral total knee arthroplasty: a cross-sectional study. Phys Ther 2014;94:1154-62.

22 Shamseer L, Moher D, Clarke M, et al. Preferred reporting items for systematic review and meta-analysis protocols (PRISMA-P) 2015 elaboration and explanation. BMJ 2015;350:97647.

23 National Institue for Health Research. PROSPERO. International prospective register of systematic reviews. Available: https://www. crd.york.ac.uk/PROSPERO/

24 Methley AM, Campbell S, Chew-Graham C, et al. PICO, PICOS and SPIDER: a comparison study of specificity and sensitivity in three search tools for qualitative systematic reviews. BMC Health Serv Res 2014; $14: 579$

25 Chamorro C, Armijo-Olivo S, De la Fuente C, et al. Absolute reliability and concurrent validity of hand held dynamometry and isokinetic dynamometry in the hip, knee and ankle joint: systematic review and meta-analysis. Open Med 2017:12:359-75.

26 Wells G, Shea B, O'Connell D, et al. The Newcastle-Ottawa Scale (NOS) for assessing the quality of case-control studies in metaanalyses. Eur J Epidemiol 2011;25:603-5.

27 Guyatt GH, Oxman AD, Vist GE, et al. GRADE: an emerging consensus on rating quality of evidence and strength of recommendations. BMJ 2008;336:924-6.

28 Vaishya R, Vijay V, Demesugh DM, et al. Surgical approaches for total knee arthroplasty. J Clin Orthop Trauma 2016;7:71-9. 